\section{MODELING FOR ACTIVE CONTROL OF COMBUSTION AND THERMALIY DRIVEN OSCILLATIONS}

\author{
F. E. C. Culick, W. H. Lin, C. C. Jahnke, and J. D. Sterling \\ Guggenheim Jet Propulsion Center \\ California Institute of Technology \\ Pasadena, California 91125
}

\begin{abstract}
Organized oscillations excited and sustained by high densities of energy release in combustion chambers have long caused serious problems in development of propulsion systems. The amplitudes often become sufficiently large to cause unacceptable structural vibrations. Because the oscillations are self-excited, they reach limiting amplitudes (limit cycles) only because of the action of nonlinear processes. Traditionally, satisfactory behavior has been achieved through a combination of trial-and-error design and testing, with control always involving passive means: geometrical modifications, changes of propellant composition, or devices to enhance dissipation of acoustic energy. Active control has been applied only to small-scale laboratory devices, but the limited success suggests the possibility of serious applications to full-scale propulsion systems. Realization of that potential rests on further experimental work, combined with deeper understanding of the mechanisms causing the oscillations and of the physical behavior of the systems. Effective design of active control systems will require faithful modeling of the relevant processes over broad frequency ranges covering the spectra of natural modes. This paper will cover the general character of the linear and nonlinear behavior of combustion systems, with special attention to acoustics and the mechanisms of excitation. The discussion is intended to supplement the paper by Doyle et al. concerned primarily with controls issues and the observed behavior of simple laboratory devices.
\end{abstract}

\section{INTRODUCTION}

The purpose of this paper is chiefly to provide a brief summary of both theoretical work and experience with combustion instabilities in propulsion systems. Due to the high densities of exothermic chemical processes in combustion chambers affording relatively low losses, conditions are favorable for the transfer of combustion energy to mechanical energy of fluid motions. Generally the combustion of reagents is stable: uncontrolled explosions or detonations are not the issue. Instabilities arise because motions of the gaseous medium interact with the burning. Burning supplies the medium comprising the combustion products and is sensitive to fluctuations of the velocity and thermodynamic properties of the flow. The situation is analogous to a feedback amplifier in which the chamber and the combustion products form the oscillator and interactions between the flow and combustion processes provide the feedback.

There is a long history of thermally driven oscillations dating back to the last century and earlier, but the problem of combustion instabilities first became a matter of practical concern about fifty years ago with the development of liquid and solid rockets. Those problems continue and must also treated in gas turbine combustors, afterburners, and ramjets. The presence of oscillations may cause structural failure, but more commonly its the vibrations that are unacceptable, producing large oscillatory accelerations of instrumentation, payloads, and guidance systems.
Combustion instabilities in practice have usually been treated by changes of the design or of the propellant system, particularly in solid rockets. Passive stabilization, including acoustic liners, baffles, and resonators, has been successfully used in liquid rockets and afterburners. Such procedures are costly and are not always entirely effective. Many operational systems have performance restricted by the necessity to avoid the appearance of oscillations.

The possibility of actively controlling combustion instabilities in liquid rockets was first suggested forty years ago, but the idea has never been applied to a practical system. Recent advances in instrumentation and computing resources suggest that successful applications should be achievable. During the past five years several groups in France, Great Britain, and the U.S. have demonstrated that oscillations can be actively controlled in small laboratory combustors operated with gaseous reagents. While the results are encouraging, the cases treated do not include some of the important features characterizing full-scale systems. For example, it appears that little work has been done in liquid-fueled systems, and no successful control has been reported. Moreover, the laboratory tests have involved relatively low-amplitude oscillations in simple geometries with modest energy densities.

Combustion instabilities most commonly occur because the system is intrinsically unstable, a consequence of the feedback mentioned above. The oscillations then reach limiting amplitudes due to the action of nonlinear processes, either gasdynamic or associated with the combustion processes. It is not unusual, however, that a linearly stable system can be pulsed into an instability by a sufficiently large disturbance.

Thus nonlinear behavior is a fundamental matter. The extent to which the control also be nonlinear is unknown; all laboratory demonstrations have been based on linear control theory with control laws accounting for a single mode of oscillation. One might argue that a sufficiently rapid system may control the oscillations before they reach amplitudes such that nonlinear behavior matters. There are at least three difficulties: it may not be possible in practice to build sufficiently fast systems; nonlinear processes may cause other modes to be unstable when one is suppressed (a phenomenon that has been observed); and finally, combustion systems exhibit obvious random behavior which may consist (to an extent unknown) of both deterministic chaotic behavior and motions induced by stochastic sources.

Experience suggests that in any case, a practical control system must be capable of handling several modes. Determining which modes to control in a particular case, and constructing the control laws, will likely depend on understanding linear and nonlinear behavior, as well as the mechanisms responsible for the instabilities. A large part of the work on combustion instabilities has been devoted to discovering and quantifying mechanisms. This has always involved a close collaboration of theory, experiment, and analysis.

As part of a larger program devoted to active control of complex fluid systems, we are engaged in investigations of the fundamental acoustics and mechanisms of combustion instabilities. Linear acoustics is for the most part well-understood: diffculties arise mainly in quantitative representation of the particular processes dominant in the system at hand. Consequently, the emphasis in this paper is on nonlinear processes. In Section 2 we briefly review the foundations and construction of an approximate analysis that has formed the basis for investigating linear and nonlinear stability of motions in combustion chambers. A few previous results will be cited to convey the idea of 
the strategies followed and to help clarify the classes of problems remaining. The way in which control theory may be connected to this framework is outlined.

Mechanisms for combustion instabilities in both liquid-fueled and solid-fueled systems are summarized in Section 3. Section 4 is a brief review of previous experimental and theoretical work on active control applied to these problems.

Understanding of the nonlinear behavior of combustion instabilities remains in a relatively modest state, chiefly for two reasons. First, experiments are expensive, and few examples of accurate data exist. Second, realistic numerical analysis is not only expensive, but impossible to carry out with confidence because of the uncertainties associated with much of the required peripheral information, notably details of combustion processes. It is for those reasons that the approximate analysis occupies such a central position in the subject, allowing both exploration of the theoretical behavior and accommodation of experimental results in a fashion not requiring great accuracy.

The remainder of the paper summarizes some recent work dealing with three subjects. Section 5 covers an application of bifurcation theory to nonlinear acoustics. Among other results, this approach allows one to assess the limits of the method of averaging used to simplify the approximate analysis. An example of applying the theory to the Rijke tube, a simple case of thermally driven oscillations, is described in Section 6. One reason for examining this problem is to aid interpretation of the experiments reported in the paper by Doyle et al. [1].

Finally, in Section 7, new work is described on analysis of one set of data taken in a laboratory combustor at Caltech. Methods developed elsewhere have been used to investigate the possible existence of deterministic chaos. The purpose is to clarify the nonlinear behavior, including nonlinear combustion processes, and eventually to make connections with the nonlinear approximate analysis. An important issue concerns the distinction between chaotic behavior and the influences of stochastic processes in combustion systems. The results described here constitute an initial investigation of that problem.

\section{FORMULATION OF THE APPROXIMATE ANALYSIS WITH PROVISION FOR ACTIVE CONTROL}

Extended discussions of the analysis may be found in several recent articles ([2]-[5]). The starting point is the complete system of conservation equations written for two-phase flow accounting for the presence of liquid or solid particulate material in the flow. After some routine manipulations and rearrangements, these equations can be put in the form

$$
\begin{aligned}
\frac{D \rho}{D t} & =\mathcal{W}+W_{c} \\
\rho \frac{D \vec{u}}{D t} & =-\nabla p+\overrightarrow{\mathcal{F}}+\overrightarrow{\mathcal{F}_{c}} \\
\rho C_{v} \frac{D T}{D t} & =-p \nabla \cdot \vec{u}+\mathcal{Q}+\mathcal{Q}_{c} \\
\frac{D p}{D t} & =-\gamma p \nabla \cdot \vec{u}+\rho+P_{c}
\end{aligned}
$$

where

$$
\begin{aligned}
\mathcal{W}+\mathcal{W} & =-\rho \nabla \cdot \vec{u}+\left[w_{c g}\right] \\
\vec{f}+\vec{F}_{c} & =\nabla \cdot \overleftrightarrow{\tau}_{v}+\left[\vec{m}_{c}+\vec{u} w_{c g}\right] \\
\mathcal{Q}+\mathcal{Q}_{c} & =\mathcal{Q}+\nabla \cdot \vec{q}-\vec{u} \cdot \nabla \cdot \stackrel{\leftarrow}{\tau}_{v}+\left[Q_{c}-e_{0} w_{c g}-\vec{u} \cdot \vec{F}_{c}\right](2.7) \\
\rho+P_{c} & =\frac{R}{C_{v}} \mathcal{Q}+\frac{R}{C_{v}}\left[\mathcal{Q}_{c}+C_{v} T w_{c g}\right]
\end{aligned}
$$

To simplify, we have dropped the influences of condensed material, a restriction easily relaxed, as discussed in the references cited above. This system of equations is completed by the equation of state for the gas,

$$
p=\rho R T
$$

There are six dependent variables: the three components of velocity $\vec{u}$, and the three thermodynamic properties: pressure $p$, density $\rho$, and temperature $T$. Correspondingly, six equations govern their evolution: (2.1), the vector equation (2.2), (2.3) or (2.4) and (2.9). Equation (2.4) for the pressure is the combination of (2.1), (2.3), and (2.9).

The specific heats $C_{v}$ and $C_{p}$ (with $\gamma=C_{p} / C_{v}$ ) and the gas constant $R$ are assumed constant. Viscous stresses are contained in the stress tensor $\overleftrightarrow{\tau}_{v}$ and $\vec{q}$ stands for internal heat conduction. Heat addition by combustion processes is represented by $Q$ and $Q_{c}$.

Control of fluid motions can be achieved only by affecting mass, momentum, and energy. The terms representing possible means of control are distinguished by subscript ( ) $)_{c}$. Corresponding terms will appear in boundary conditions, so this formulation contains all possible schemes for control. For example, a speaker emitting acoustic waves may be modeled as an oscillatory source of mass $\left(w_{c q}\right)$ at the boundary, having zero average value.

These equations form the basis for all analysis of combustion instabilities. To investigate particular problems it is necessary to model various physical processes, such as those producing heat addition and, short of complete numerical analysis, the equations must be simplified. First, all dependent variables are written as sums of average values and fluctuations; we assume here that the average values are independent of time. After substituting those forms in the conservation equations, we have several possible strategies to follow. Here we retain terms up to first order in the mean flow and fluctuations (i.e., of order $\bar{u} u^{\prime}, \bar{u} p^{\prime} \ldots$ ) and terms quadratic in the fluctuations (of order $\left.u^{\prime 2}, p^{\prime 2}, p^{\prime} u^{\prime}, \ldots\right)$. This process produces equations nonlinear in the fluctuating variables. One way of treating that system is by iteration, a method leading to linear sets of equations for the successive approximations. The procedure followed here is based on approximate forms of the nonlinear equations, a strategy permitting more direct analysis of the global nonlinear behavior.

The next step in conversion of the partial differential equations to ordinary equations by applying a form of Galerkin's method, founded on expansion of the dependent variables in normal modes having time-varying coefficients. Spatial averaging gives a set of coupled nonlinear ordinary differential equations which form the basis of the approximate method.

It has long been known (the most complete discussion has been given by Chu and Kovasznay [6]) that any small amplitude disturbance may be synthesized of three modes of motion: acoustical, vortical and entropic. Entropy waves move with the fluid speed and have practically no pressure changes: they may be regarded as 'hot' spots of fluid carried by the flow and diffusing due to heat conduction. Vortical or shear waves are disturbances of vorticity, also carried by the fluid motion, have no pressure fluctuations and dissipate through the action of viscous stresses. By far most important for combustion instabilities are acoustic waves, propagating at the speed of sound and characterized by fluctuations of pressure, temperature, density, and velocity, all of comparable size, but having negligible vorticity or entropy changes. These three kinds of waves propagate independently in the linear approximation and can interact only at boundaries or in regions of nonuniform burning. 
Independence of propagation does not obtain in nonlinear motions, but coupling between the three sorts of waves tends to remain small and we may ignore that possibility in first approximation. That assumption has the fundamentally important consequences that we can focus attention on acoustic waves. Hence we can build a framework for investigating combustion instabilities by treating the pressure fluctuations as the primary independent variable and ignoring entropy and vorticity disturbances except as they may arise in special ways. We could, in principle, work with density, temperature, or velocity fluctuations, but it is easier to deal with the pressure, which is also the only variable usually measured when combustion instabilities occur.

These remarks describe incompletely the foundation of the analysis used in the remainder of this paper. First a wave equation for pressure fluctuation is formed:

$$
\nabla^{2} p^{\prime}-\frac{1}{\bar{a}^{2}} \frac{\partial^{2} p^{\prime}}{\partial t^{2}}=h+h_{c}
$$

where $\bar{a}^{2}=\gamma \bar{p} / \bar{\rho}$. The boundary condition is set on the gradient of $\boldsymbol{p}^{\prime}$ by taking the scalar product of the outward normal vector with the momentum equation, giving

$$
\hat{n} \cdot \nabla p^{\prime}=-f-f_{c}
$$

The definitions of $h, h_{c}, f$, and $f_{c}$ are produced in this process. At this point, the details are unimportant but it is worth noting that the contributions representing possible realizations of active control are

$$
h_{c}=\nabla \cdot \mathcal{F}_{c}^{\prime}=\frac{1}{\bar{a}^{2}} \frac{\partial P_{c}^{\prime}}{\partial t} ; \quad f_{c}=-\mathcal{F}_{c}^{\prime} \cdot \hat{n}
$$

To apply Galerkin's method we need a set of orthogonal basis functions $\psi_{n}$. The functions are to be found as the eigenfunctions for the acoustic problem solved in the actual geometry being considered. For many problems, good results are obtained if the functions are taken to be the normal modes for the classical acoustics problem having no perturbations in the volume or at the boundary. Then the $\psi_{n}$ satisfy

$$
\nabla^{2} \psi_{n}+k_{n}^{2} \psi_{n}=0 ; \quad \hat{n} \cdot \nabla \psi_{n}=0
$$

where the wavenumber for the $n^{\text {th }}$ classical mode is $k_{n}=\omega_{n} / \bar{a}$. It is essential that the speed of sound have value as close as possible to that in the actual problem with combustion and flow.

The matter of constructing the $\psi_{n}$ is more complicated if there are significant perturbations at the boundary, as for a choked nozzle with high inlet Mach number, or if the temperature field is highly non-uniform. A second case arises in problems where significant areas of flame sheets exist, or heat is added at well-defined surfaces. An example is analyzed here in Section 6 . To illustrate the general procedure we assume now that the basis functions satisfy $(2.13 a, b)$. Then the pressure and velocity fluctuations are expanded in the following series

$$
\begin{aligned}
& p^{\prime}(\vec{r}, t)=\bar{p} \sum_{i=1}^{\infty} \eta_{i}(t) \psi_{i}(\vec{r}) \\
& u^{\prime}(\vec{r}, t)=\sum_{i=1}^{\infty} \frac{\eta_{i}(t)}{\bar{\gamma} k_{i}^{2}} \nabla \psi_{i}(\vec{r})
\end{aligned}
$$

Substitution in the left-hand side of (2.10), multiplication by $\psi_{n}$, integration over the chamber, and use of Green's theorem leads to the coupled set of equations for the amplitudes $\eta_{n}(t)$ :

$$
\frac{d^{2} \eta_{n}}{d t^{2}}+\omega_{n}^{2} \eta_{n}=F_{n}+F_{c n}
$$

where

$$
\begin{aligned}
F_{n} & =-\frac{\bar{a}^{2}}{\bar{p} E_{n}^{2}}\left\{\int h \psi_{n} d V+\iint f \psi_{n} d S\right\} \\
F_{c n} & =-\frac{\bar{a}^{2}}{\bar{p} \bar{E}_{n}^{2}}\left\{\int h_{c} \Psi_{n} d r+\iint f_{c} \psi_{n} d S\right\}
\end{aligned}
$$

and

$$
E_{n}^{2}=\int \psi_{n}^{2} d V
$$

Solution to the system (2.15) requires that $F_{n}$ and $F_{\text {on }}$ be expressed as functions of the amplitudes and their derivatives: thus the general problem has been reduced to a much narrower problem of modeling the dominant physical processes. The gas dynamics can be represented accurately, leading to equations containing both interactions between the mean flow and the fluctuations, and nonlinear coupling of the fluctuations themselves.

The source terms $F^{\prime}, F_{c}^{\prime}, P^{\prime}$, and $P_{c}^{\prime}$ contain, in addition to internal viscous stresses and heat conduction, all processes that cause combustion instabilities and define their special attributes. Viscous stresses and heat conduction are accurately known. Hence the strategy in the approximate analysis is to account for the gasdynamics, viscous stresses and heat conduction according to known results, and concentrate in modeling those processes that, because they are not well-understood, can be handled only approximately. Experimental results are eventually the only means for determining the truth of the modeling.

Linear processes always lead to terms proportional to $\eta$ or $\dot{\eta}$ on the right-hand side of (2.15), having the form $D \dot{\eta}+E \eta$. It happens that within the approximations used here, nonlinear gasdynamics produces terms quadratic in $\eta$ and $\dot{\eta}$ but no cross-coupling involving $\eta \dot{\eta}$. With these contributions shown explicitly, the system (2.15) is

$$
\begin{aligned}
& \frac{d^{2} \eta_{n}}{d t^{2}}+\omega_{n}^{2} \eta_{n}= \\
& -\sum_{i=1}^{\infty}\left[D_{n i} \dot{\eta}_{i}+E_{n i} \eta_{i}\right]-\sum_{i=1}^{\infty} \sum_{j=1}^{\infty}\left[A_{n i j} \dot{\eta}_{i} \dot{\eta}_{j}+B_{n i j} \eta_{i} \eta_{j}\right]+F_{n}+F_{c n}
\end{aligned}
$$

where now we mean that $F_{n}$ and $F_{\text {cn }}$ contain linear and nonlinear processes not arising from the particular contributions mentioned above. Hence, within this analysis, modeling additional flow and combustion processes, and representing control mechanisms, must eventually come down to constructing special forms for $F_{n}$ and $F_{c n}$. Some of those results may of course simply provide more linear and quadratic terms like those shown.

Much effort for practical applications has been spent on linear stability of motions, defined by the set of equations

$$
\frac{d^{2} \eta_{n}}{d t^{2}}+\omega_{n}^{2} \eta_{n}=-\sum\left[D_{n i} \dot{\eta}_{i}+E_{n i} \eta_{i}\right]
$$

Linear coupling is generally absent in these problems, so the system (2.19) becomes a set of independent equations for each of the normal modes:

$$
\frac{d^{2} \eta_{n}}{d t^{2}}+D_{n n} \frac{d \eta_{n}}{d t}+\left(\omega_{n}^{2}+E_{n n}\right) \eta_{n}=0
$$

The solution is

$$
\eta_{n}(t)=\hat{\eta} e^{-\frac{D_{n n}}{3} t} \cos \left[\sqrt{\omega_{n}^{2}+E_{n n}} t+\phi_{n}\right]
$$


and the stability boundary for the $n^{\text {th }}$ mode is defined by $D_{n n}=$ 0 . In practice, the frequency shift, approximately $E_{n} / 2 \omega_{n}$, is small and usually cannot be used to provide useful information from experimental data. In contrast, determination of $D_{n n}$ both theoretically and experimentally has been the central issue in studying combustion instabilities. There is a large amount of literature dealing with this matter (e.g., $[2]-[4],[7],[8],[9])$ and we shall not pursue the subject here.

The terms on the right-hand side of (2.18) are often small, and may be justifiably regarded as perturbations. Hence timeaveraging is a useful means of simplifying the equations, leading to a set of first order equations useful for both applications and for theoretical work. The idea is based on the observation that most combustion instabilities are oscillations having slowly varying amplitudes and phases. Then $\eta_{n}(t)$ may be realistically represented in the form

$$
\begin{aligned}
\eta_{n}(t) & =r_{n}(t) \sin \left(\omega_{n} t+\phi_{n}(t)\right) \\
& =A_{n}(t) \sin \omega_{n} t+B_{n}(t) \cos \omega_{n} t
\end{aligned}
$$

Substitution in (2.15) and time-averaging over an interval $(t, t+$ r) gives first-order equations for $A_{n}$ and $B_{n}$.

To obtain specific results, the expansions $(2.14 a, b)$ must be truncated. Because the higher modes are strongly attenuated (usually only one or two of the lowest modes are unstable) quite approximations may be obtained with a surprisingly small number of modes. When only two modes are considered [10], the equations are

$$
\begin{aligned}
& \frac{d A_{1}}{d t}=\alpha_{1} A_{1}+\theta_{1} B_{1}-\beta\left(A_{1} A_{2}-B_{1} B_{2}\right) \\
& \frac{d B_{1}}{d t}=\alpha_{1} B_{1}-\theta_{1} A_{1}+\beta\left(B_{1} A_{2}-A_{1} B_{2}\right) \\
& \frac{d A_{2}}{d t}=\alpha_{2} A_{2}+\theta_{2} B_{2}+\beta\left(A_{1}^{2}-B_{1}^{2}\right) \\
& \frac{d B_{2}}{d t}=\alpha_{2} B_{2}=\theta_{2} A_{2}+2 \beta A_{1} B_{1}
\end{aligned}
$$

where $\beta=\omega_{1}(\gamma+1) / 8 \gamma$. These equations can be solved exactly to give the conditions for existence and stability of limit cycles $([5],[10])$. Corresponding results have been obtained for the lowest modes in a cylindrical chamber [5]. The results seem to be accurate over quite broad conditions, a conclusion established by comparison with numerical solutions to the conservation equations. Figure 2.1 shows the waveforms in a limit cycle; five modes being accounted for in the approximate analysis. The frequencies of the modes are within $3 \%$ of those inferred from spectral analysis of the numerical results. Good agreement is obtained for the first three modes; larger differences arise for the fourth and fifth modes because truncation of the expansion excludes transfer of energy to higher modes $(n>5)$, causing those retained to reach larger amplitudes.

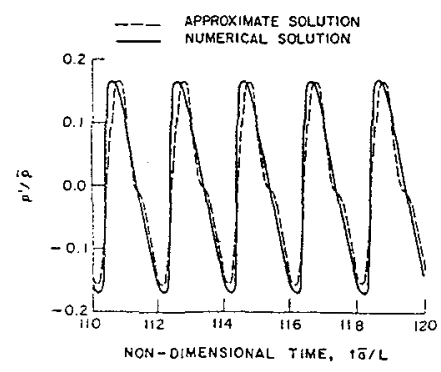

Figure 2.1

The main purpose of this section is to describe briefly an approximate analysis suitable as the basis for modeling both linear and nonlinear behavior of combustion instabilities. Experience has shown that the results are quite good under broad practical conditions. The theoretical framework seems to be particularly attractive for investigating applications of active control.

\section{MECHANISMS OF COMBUSTION INSTABILITIES}

There are two main sources of energy available to drive combustion instabilities: the burning processes themselves and the flow field of the combustion products. Ultimately, of course chemical processes are the source, but it is convenient to make this division. Interaction between the combustion chamber, the propellant supply system, and the supporting structure is also an important cause of instabilities, mainly in large rockets. Often called the POGO instability, the problem is well-understood, and can be solved in practice. We shall not consider it further in this paper although a form of this instability was the basis for the first proposal of active control by Tsien [11] and it remains as a possible application.

There are several forms of coupling between the average and unsteady flow fields, some of which are explicitly displayed in the general conservation equations. However, both in laboratory devices and in operational combustors, oscillations have occasionally been found to be caused by flow separation and shedding of large-scale vortices [12]-[14], a phenomenon not apparent from the equations. Vortex shedding may excite oscillations either by purely fluid-mechanical interactions or through influences on combustion processes. The first leads to relatively weak instabilities because the available mechanical energy is relatively small. In contrast, when combustion is involved, substantial oscillations may be produced.

Broadly, vortices may entrain reactants and owing to chemical or mixing time delays, combustion may subsequently occur at times and in spatial locations favorable to destabilizing an acoustic mode. Examples of this behavior have been known for more than thirty-five years, but most of the work on the problem has been accomplished during the past decade (see [2], [3], and [15] for summaries and references to the original works).

A possible but less important mechanism for exciting combustion instabilities involves convective waves. If a region of high temperature passes into a supersonic nozzle, interaction with the region of large nonuniform flow produces an acoustic wave which will then propagate upstream. Then the action of the acoustic wave on combustion processes may generate regions of nonuniform temperature developing into convective waves. Thus a feedback loop exists within the chamber, sustaining oscillations. Simple theoretical models support this view, and such modes of oscillation have been identified also in numerical analysis of unsteady motions in dump combustors [15]. It is likely that instabilities excited by this mechanism have occurred in laboratory combustors, but the evidence is scarce and inconclusive.

By far the most important mechanisms responsible for combustion instabilities are associated with injection of the propellants, formation of liquid droplets, and combustion of the reacting gases. The processes involved are extremely complicated on both the macroscopic and microscopic levels and are not thoroughly understood. Any success that has been achieved in suppressing instabilities by affecting those processes has been largely by trial and error, a strategy contributing significantly to development costs, particularly of afterburners and liquid propellant rockets. Reference [7] contains reviews of most of the work on liquid rockets accomplished prior to 1970 , motivated 
largely by the requirements of the APOLLO program. Although a few reports have been published on experience with afterburners, the bulk of the information is proprietary or classified.

It seems presently that the most promising applications of active control are for liquid-fueled systems. In view of the relatively incomplete state of understanding, considerable laboratory and theoretical work remains to ensure success.

\section{PREVIOUS WORK ON ACTIVE CONTROL OF COMBUSTION INSTABILITIES}

Tsien's [11] idea for active control consisted in actuating a capacitance in the fuel supply system. This approach allows control of a time delay in the in system comprising the combustion chamber and the propellant supply. It's a method that will work for low-frequency oscillations called "chugging" or, when structural vibrations are also involved, "POGO" instabilities. The problem is particularly troublesome in large liquid rocket systems, but in practice, passive means have proved adequate for more-or-less satisfactory solution. Eventually active control may provide a better method for solution but there seems to be no current work on the problem.

True active control of instabilities was first demonstrated in a series of works carried out at Cambridge University under the direction of Dowling ([16]-[18] and other references cited there). Only gas-fueled laboratory devices have been studied, with control exerted by controlling loudspeakers, a vibrating plug in the inlet nozzle, or a secondary fuel injector. The instabilities consist of longitudinal nodes excited by coupling with a narrow flame zone stabilized in a long duct. This configuration is intended to simulate one element of the sort of injection system used in afterburners.

Lang, Poinsot, and Candel [19] and Poinsot et al. [20] have reported similar work on a laboratory device using loudspeakers. A group at General Electric [21] has also tested a duct burner of the same sort, again using gaseous fuels and exercising control by generating acoustic waves. They have informally noted lack of success when they tried modulating the supply of liquid fuel.

In the latest report of a program concerned with control of instabilities excited by vortex shedding, Wilson et al. [22] have successfully reduced the amplitude of oscillation by modulating a secondary fuel supply injected in the region where the vortices are initiated. The tests are interesting for several reasons. First, the geometry is a faithful simulation of that common to fullscale ramjet engines, and the power generated in the combustor ( 1 megawatt) is substantially higher than in the devices cited above. Second, experiments have been conducted with sensing fluctuations of the pressure or of radiation by a combustion product, the latter results not reported in the reference. Use of various means of flow visualization in this continuing work offers some promise of clarifying the mechanisms, thereby providing a basis for more effective control.

While the works cited suggest the possibilities of active control, they constitute only a beginning. In all cases, only a single sensor and a single actuator were used, and the control strategy was limited to a single mode. Relatively low-amplitude instabilities have been treated and suppression has been achieved only in part of the possible operating range of fuel/air ratio. Complete suppression has rarely been observed and so results have been reported for instabilities initiated by impulsive disturbances. Essentially no advantage has been taken of the results provided by modern control theory.

Yang, Sinha, and Fung [23] have given the only discussion of control theory applied to combustion instabilities. Their approach begins with the approximate analysis described here in
Section 2 , using the amplitudes and velocities $(\eta, \dot{\eta})$ of the nodal oscillators as the state variables. The results follow from application of the theory described in texts such as that by Franklin, Powell, and Emami-Naeini [24]. They show computer simulations for the longitudinal models with simple representation of the actuation and no detailed modeling of the possible mechanisms. Nonlinear behavior and uncertainties in the characteristics of the system have not been considered.

\section{APPLICATION OF BIFURCATION THEORY TO NONIINEAR ACOUSTICS}

Bifurcation theory and continuation methods will be used to analyze the approximation to equation (2.15) that includes linear contributions from the combustion processes, gas/particle interactions, mean flow and boundary conditions, and nonlinear contributions from second-order gas dynamics [10]. After rescaling time, $t \mapsto \omega_{1} t$, and defining the new variable

$$
\varsigma_{n}=\dot{\eta}_{n},
$$

the equation for longitudinal modes in a cylindrical combustion chamber has the form

$$
\begin{aligned}
\dot{\eta}_{n}= & \varsigma_{n}, \\
\dot{\zeta}_{n}= & -n^{2} \eta_{n}+2 \alpha_{n} \zeta_{n}+2 n \theta_{n} \eta_{n} \\
& -\sum_{i=1}^{n-1}\left(C_{n i}^{(1)} \varsigma_{i} \zeta_{n-i}+D_{n i}^{(1)} \eta_{i} \eta_{n-i}\right) \\
& -2 \sum_{i=1}^{\infty}\left(C_{n i}^{(2)} \varsigma_{i} \zeta_{n+i}+D_{n i}^{(2)} \eta_{i} \eta_{n+i}\right)
\end{aligned}
$$

where $C_{n i}^{(1)}, C_{n i}^{(2)}, D_{n i}^{(1)}$, and $D_{n i}^{(2)}$ are constants depending on the ratio of specific heats of the gas.

The modal expansion is truncated at some value of $n$ to make the problem tractable. In order to make analytical analysis of the equations possible, the expansion has typically been truncated at two modes. This results in a fourth-order system, which is analytically tractable, and also contains the important physical characteristic of energy transport between modes. Energy is typically transported to higher-frequency modes, so the truncation at two modes is only valid for an unstable first mode (i.e., $\alpha_{1}>0$ ). The results presented here will be for a system that has an unstable first mode. The values of the linear parameters used in this analysis were obtained from [10].

The truncated system is also averaged in time to further simplify the analysis [10], [25]. Theoretically the Averaging Theorem is valid for systems of the form

$$
\dot{x}=\epsilon f(x, \mu, \epsilon),
$$

where $\epsilon$ is a small parameter. Equation (5.2) only has this form when $\eta$ and $\zeta$ are small so one would expect the results from the averaged system to differ from the results of the original system when $\eta$ and $s$ become large.

In this work continuation methods are used to calculate the periodic orbits of (5.2). Continuation methods are numerical techniques for determining the steady states or limit cycles of a system of ordinary differential equations as a function of one of the parameters of the system [26]. In the results presented below, the parameter of interest is the linear damping of the first mode, $\alpha_{1}$. The use of continuation methods has made it possible to analyze higher-dimensional systems and determine the validity of time averaging and truncation to two modes during the analysis of combustion instabilities. 
Figure 5.1 shows a comparison of the results obtained by applying the continuation technique to (5.2) and by analyzing the averaged system.

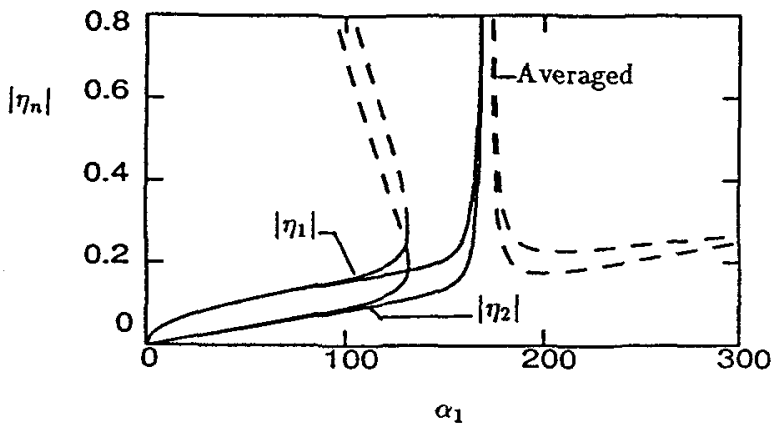

Figure 5.1: Maximum amplitude of acoustic modes in limit cycle for second-order two-mode approximation. - Stable, - - - Unstable

The figure shows that the results of the averaged system are essentially the same as the results of the original system when the amplitude of the limit cycle is small. The results start to diverge as the amplitude of the limit cycle increases and the stability boundary predicted by the two methods are substantially different. For the averaged system the stability boundary is given by $\alpha_{2} / \alpha_{1}=-2[10]$. It is important to note however, that the amplitude of the limit cycles of the averaged system are

$$
\begin{aligned}
& \left|\eta_{1}\right|=\frac{8 \gamma}{(\gamma+1) \omega_{1}} \sqrt{-\alpha_{1} \alpha_{2}\left(1+\beta^{2}\right)} \\
& \left|\eta_{2}\right|=\frac{8 \gamma}{(\gamma+1) \omega_{1}} \sqrt{\alpha_{1}^{2}\left(1+\beta^{2}\right)}
\end{aligned}
$$

where

$$
\beta=\frac{\theta_{2}-2 \theta_{1}}{\alpha_{2}+2 \alpha_{1}}
$$

Thus, as $\alpha_{2} / \alpha_{1} \mapsto-2$, the amplitude of the limit cycle approaches infinity. This would lead one to expect that the predictions of the averaged system are not correct when $\alpha_{2} / \alpha_{1}$ is close to -2 . Figure 5.2 shows a comparison of the stability boundaries predicted by the original and averaged equations.

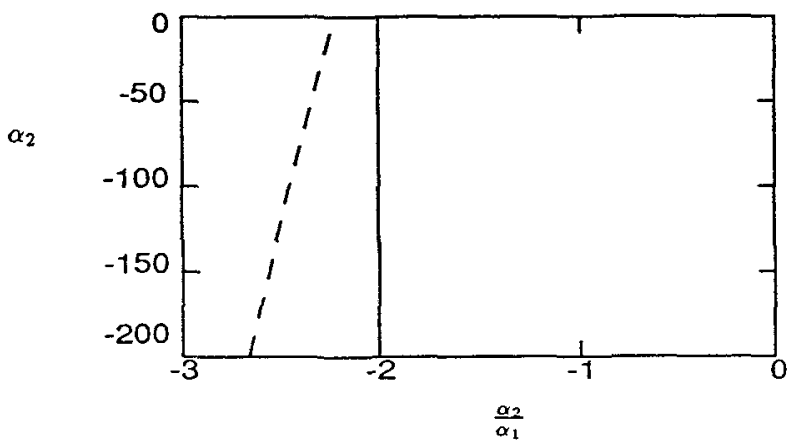

Figure 5.2: Stability boundaries for two-mode secondorder equations; $\theta_{1}=0.0, \theta_{2}=10.0, \omega_{1}=900 \mathrm{~Hz}$.

Averaged Equations, - - Original Equations

Figures 5.1 and 5.2 show that one must be careful when using the averaged equations to analyze combustion instabilities. The averaged equations give accurate results when the amplitude of the limit cycle is small and when the ratio $\alpha_{2} / \alpha_{1}$ is greater than -2 . Thus, the applicability of the averaged equations will depend on the individual combustion system, which will have specific values of $\alpha_{1}$ and $\alpha_{2}$. The values of $\theta_{1}$ and $\theta_{2}$ may also affect the validity of the averaged equations, but this has not been studied in detail at this point.

Another issue which must be studied is the effect of truncating the system at a finite number of modes. In fluid mechanical systems energy is transported from low-frequency modes to higher-frequency modes where it is dissipated. By truncating the system at a relatively small number of modes this flow of energy to higher-order modes is clearly disrupted. Previously the effect of including more modes in the system has only been studied through numerical simulations because of the difficulty of analyzing systems of six or more equations [10].

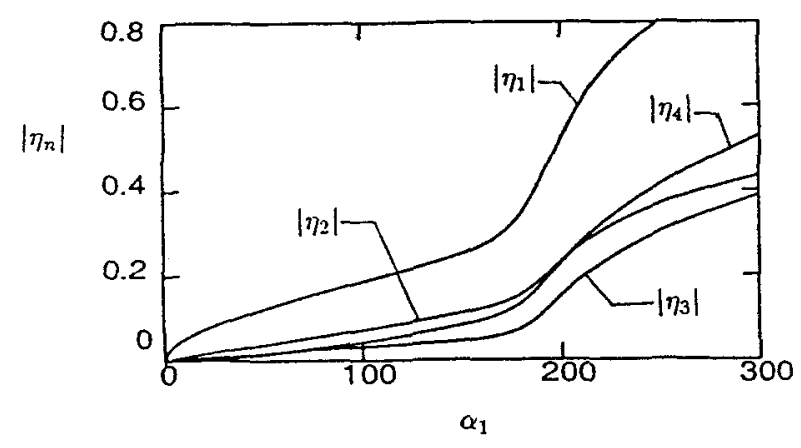

Figure 5.3: Maximum amplitude of acoustic modes in limit cycle for second-order, four-mode approximation.

$$
\text { Stable, - - Unstable }
$$

Continuation methods make it possible to analyze the limit cycles of higher-order systems. Figure 5.3 shows the amplitude of the limit cycle of the four-mode approximation for the same case shown in Figure 5.1. The main difference between the twoand four-mode approximations is the absence of a stability boundary in the four-mode approximation. There is a rapid increase in the magnitude of the limit cycle as the ratio $\alpha_{2} / \alpha_{1}$ nears -2 , but there is no instability. It is also interesting to note that the higher modes, $\eta_{3}$ and $\eta_{4}$, are smaller than the first and second modes when $\alpha_{1}$ is small, but when $\alpha_{1}$ is larger than 100 , the third and fourth modes are almost the same size as the second mode. For $\alpha_{1}$ greater than 200, the amplitude of the fourth mode becomes larger than the amplitude of the second mode.

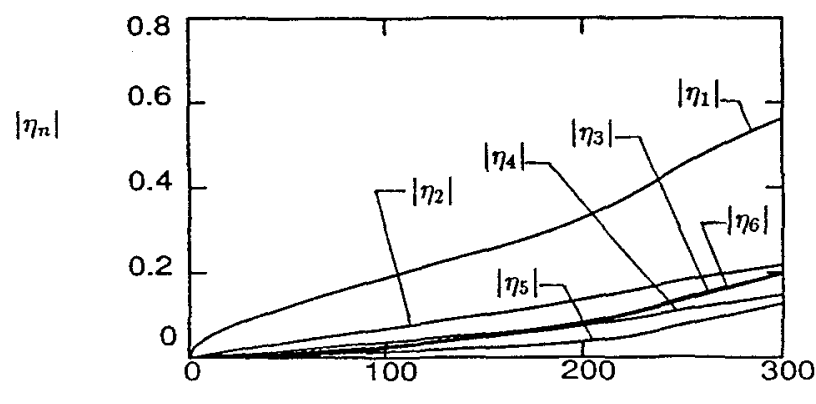

$\alpha_{1}$

Figure 5.4: Maximum amplitude of acoustic modes in limit cycle for second-order, six-mode approximation.

$$
\text { - Stable, - - Unstable }
$$

Figure $5 . \dot{4}$ shows the results for a system of six modes. The results for four- and six-mode systems are essentially the same 
when $\alpha_{1}$ is less than 100. For values of $\alpha_{1}$ greater than 100, the amplitude of the first and second modes in the limit cycle are much larger in the four-mode system than the six-mode system. This is a result of the fact that more stable modes are available to dissipate the energy generated by the unstable first mode in the six-mode approximation than in the four-mode approximation.

These results suggest that, depending on the linear damping parameters of a particular combustion system, it may be necessary to use more than two modes to analyze the system. The results certainly show that caution must be exercised when determining the stability domain of a truncated system of modes, as adding more modes may cause the stability boundary to disappear. One must also be sure to consider the limitations of the averaged system when interpreting results from the averaged system. In particular, it is important to make sure that the amplitude of the limit cycle is small in the region of interest.

\section{ANALYSIS OF THE RIJKE TUBE}

The Rijke tube offers the simplest example of thermally driven oscillations and is an attractive illustration of some of the characteristics of combustion instabilities. Figure 6.1 shows the physical situation. A uniform tube is fitted with a heater, the simplest being coiled wire carrying an electric current, or in a more complicated form a flat flame is stabilized in a transverse plane. Under appropriate conditions, which include suitable placement of the heater

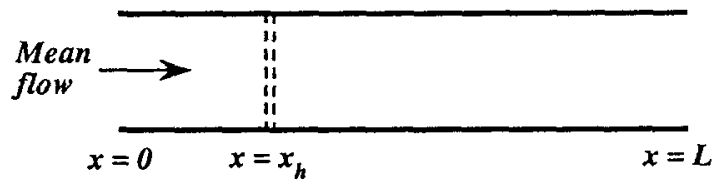

Figure 6.1: Rijke tube with heat source at $x=x_{h}$.

and the presence of an average flow, the heat source will excite longitudinal modes of acoustic vibration and the tube emits audible sound which may reach quite large amplitudes, over 100 $\mathrm{dB}$.

Rayleigh [27] was motivated by observations of the Rijke tube, among other phenomena, to formulate his "criterion" that heat addition in phase with the pressure oscillation encourages the motion. In its original form, Rayleigh's criterion is an accurate qualitative statement, but for detailed analysis of experimental data, it is usually more useful to have an explicit quantitative form, such as given by Chu [28] and Culick [29]. If the idea is developed completely, and all energy losses and gains are accounted for, one finds that the general form of the criterion is precisely equivalent to the statement deduced from linear stability analysis that the growth constant $\alpha$ (in $e^{\alpha t}$ ) must be positive for a wave to be unstable.

Carrier [30] was first to give a detailed analysis of the Rijke tube based on solution to the conservation equations. Perhaps the most significant part of that work is Carrier's calculation of the influence of the fluctuating acoustic velocity on the heat addition from a heated metallic ribbon to the flow. The result can be expressed as a transfer function from velocity to heat release, a representation of the mechanism for exciting oscillations in this problem. Merk [31], [32] later treated the problem in similar fashion and deduced two formulas for the transfer function based on slightly different models of the heat transfer process. The following remarks show how this representation of the mechanism is incorporated in the approximate analysis.

We assume that the flow is one-dimensional, and to emphasize the influences of the heat addition we ignore all influences of viscous effects, the mean flow and acoustic radiation at the ends. One consequence of these assumptions is that we do not account for the increase of velocity due to the decrease of density in flow past the heater. A complete analysis to be reported in later work will include those effects. The fluctuating heat release is represented as

$$
Q^{\prime}=T u^{\prime} \delta\left(x-x_{h}\right)
$$

for a source concentrated at $x=x_{h}$. The transfer function $T$ is complex. We suppose that control is exercised by a speaker modeled as an oscillating source of mass, $w_{c g}$, at $x=x_{c}$,

$$
w_{\mathrm{cg}}=u_{\mathrm{c}} \delta\left(x-x_{c}\right)
$$

Equation (2.27) then becomes

$$
\frac{d^{2} \eta_{n}}{d t^{2}}+\omega_{n}^{2} \eta_{n}=\frac{1}{\bar{p} E_{n}^{2}}\left[(\gamma-1) \psi_{n}\left(x_{h}\right) T \frac{\partial u^{\prime}}{\partial t}\left(x_{h}\right)+R \bar{T} \psi_{n}\left(x_{c}\right) \frac{d u_{c}}{d t}\right]
$$

Substituting (2.26b) into (6.3), and assuming no linear coupling between modes, leads to

$$
\begin{aligned}
\frac{d^{2} \eta_{n}}{d t^{2}} & +\omega_{n}^{2} \eta_{n} \\
& =\frac{1}{\bar{p} E_{n}^{2}}\left[\frac{\gamma-1}{\gamma k_{n}^{2}} \psi_{n}\left(x_{h}\right) \frac{d}{d x} \psi_{n}\left(x_{h}\right) T \frac{d^{2} \eta_{n}}{d t^{2}}+R \vec{T} \psi_{n}\left(x_{c}\right) \frac{d u_{c}}{d t}\right]
\end{aligned}
$$

We assume that the right-hand side is small, so we can appeal to the assumption used as the basis for time-averaging in Section 2 , that $\eta_{n}(t)$ is an oscillation having slowly varying amplitude and phase. Since $T$ is already assumed small, in the first term on the right-hand side we may write $\ddot{\eta}_{n} \approx-\omega_{n}^{2} \eta_{n}$, and we have, with $\omega_{n}^{2}=\bar{a}^{2} k_{n}^{2}$,

$$
\begin{aligned}
& \frac{d^{2} \eta_{n}}{d t^{2}}+\omega_{n}^{2} \eta_{n} \\
& =\frac{1}{\bar{p} E_{n}^{2}}\left[\left(\bar{a}^{2} \frac{\gamma-1}{\gamma} \psi_{n}\left(x_{h}\right) \frac{d}{d x} \psi_{n}\left(x_{h}\right) T \eta_{n}\right)+R \bar{T} \psi_{n}\left(x_{c}\right) \frac{d u_{c}}{d t}\right]
\end{aligned}
$$

This equation has the same form as (2.32), the second term representing control with mass addition (the speaker). The first term, being complex, represents both a frequency shift and damping due to the energy source. This can be shown most directly by writing $\eta_{n}(t)=\hat{\eta}_{n} e^{-i(\omega+i \alpha) t}$ where $\omega=\omega_{n}+\delta \omega$ is the shifted frequency and $\alpha$ is the growth constant of the $n^{t h}$ mode. Then

$$
\begin{aligned}
T \eta_{n} & =\left(T_{r}+i T_{i}\right) \hat{\eta}_{n} e^{-i(\omega+i \alpha) t} \\
& =\left(T_{r}+\frac{T_{i}}{\omega} i \omega\right) \hat{\eta}_{n} e^{-i(\omega+i \alpha) t} \\
& \approx T_{r} \eta_{n}+\frac{T_{i}}{\omega_{n}} \frac{d \eta_{n}}{d t}
\end{aligned}
$$


where we neglect terms of second order in small quantities. Hence the first term has the form

$$
\frac{\bar{a}^{2}}{\bar{p} E_{n}^{2}} \frac{\gamma-1}{\gamma} \psi_{n}\left(x_{h}\right) \frac{d \psi_{n}}{d x}\left(T_{r} \eta_{n}+\frac{T_{i}}{\omega_{n}} \frac{d \eta_{n}}{d t}\right)=-D_{n n} \dot{\eta}_{n}-E_{n n} \eta_{n}
$$

with

$$
\begin{aligned}
& D_{n n}=-\frac{\bar{a}^{2} / \omega_{n}}{\bar{p} E_{n}^{2}} \frac{\gamma-1}{\gamma} \psi_{n}\left(x_{h}\right) \frac{d \psi_{n}}{d x} T_{i} \\
& E_{n n}=-\frac{\bar{a}^{2}}{\bar{p} E_{n}^{2}} \frac{\gamma-1}{\gamma} \psi_{n}\left(x_{h}\right) \frac{d \psi_{n}}{d x} T_{r}
\end{aligned}
$$

Hence, according to Equation (2.33), the growth constant is

$$
\alpha=\frac{\bar{a}^{2} / \omega_{n}}{2 \bar{p} E_{n}^{2}} \frac{\gamma-1}{\gamma} \psi_{n}\left(x_{h}\right) \frac{d \psi_{n}}{d x} T_{i}
$$

Linear stability depends on the imaginary part of the transfer function. Of course, the other contributions mentioned above and ignored in these calculations will also contribute to stability.

Consider, for example, the fundamental mode for a tube open at both ends, so the pressure fluctuation vanishes at $x=$ $0, L$ :

$$
\psi_{1}=\sin \pi \frac{x}{L}
$$

The wave number is $k_{1}=\pi / L=\omega_{1} / \bar{a}$, and substitution in (6.9) gives

$$
\alpha=\frac{\bar{a}^{2}}{4 \bar{p} E_{n}^{2}} \frac{\gamma-1}{\gamma} \sin \left(2 \pi \frac{x_{h}}{L}\right) T_{i}
$$

Hence $\alpha$ is positive and a small disturbance in the fundamental mode is unstable if $0<x_{h}<L / 2$. This is a well-known result obtained with Rayleigh's criterion and of course found as well by Carrier and Merk.

Merk [32] has given the three formulas for the transfer function:

$$
\begin{aligned}
& T=\frac{1 / 2}{1-i \omega \tau} \\
& T=i \frac{P e}{2 \tilde{\omega}} \frac{\ln (1-4 i \tilde{\omega} / P e)^{\frac{1}{2}}}{\ln (8 / P e)-C-\ln (1-4 i \tilde{\omega} / P e)^{\frac{1}{2}}} \\
& T=\frac{i}{2 \tilde{\omega}}\left[e^{i \tilde{\omega}}-1+(-i \pi \tilde{\omega})^{\frac{1}{2}} \operatorname{erf}\left[(-i \tilde{\omega})^{\frac{1}{2}}\right]\right]
\end{aligned}
$$

The first is for a cylinder at high Reynolds numbers, so viscous effects occur in a boundary layer; the second is for a cylinder at low Reynolds numbers in the range of the Oseen approximation; and the last is for a ribbon for large Peclet numbers, $P e=$ $\operatorname{RePr} \gg>1$, where $\operatorname{Re}=\rho b \bar{u} / \mu, \operatorname{Pr}=\mu C_{p} / k$, and $b$ is the width of the ribbon. For all three cases, $T_{i}$ is positive, implying that the oscillating heat transfer lags the velocity fluctuation. The time constant $\tau$ in $(6.12 a)$ has the form

$$
\tau=\frac{d}{\bar{u}} g(R e, P e)
$$

where $R e=\rho d \bar{u} / \mu$ is Reynolds number, $d$ is the diameter of the cylinder, and $g(R e, P e)$ is a function of $R e$ and $P e$. In $(6.12 b)$, $C$ is Euler's constant and $\tilde{\omega}=\omega d / \bar{u}$.

The remarks here are intended only to show how the analysis constructed in Section 2 can be applied to a useful example. Realistic results require that all processes be accounted for.
Moreover, the modeling of the heat source discussed here is oversimplified, since rectification is ignored. The form (6.1) is valid only if the amplitude of the fluctuation is less than the mean velocity. The reason is that the heat transfer depends only on the magnitude of the velocity past the surface, not the sense. If, for example, a cylinder is exposed to an oscillating velocity normal to its axis, with no mean flow, the frequency of the oscillating heat transfer is twice that of the velocity. That is the reason that an average flow is required for the Rijke tube to work.

\section{DYNAMICAL SYSTEM CHARACTERIZATION OF PRESSURE OSCILLATIONS}

Several acoustic modes may participate in the pressure oscillations associated with combustion instabilities for several reasons. For example, as mentioned in Section 1, the active control of one acoustic mode may result in the appearance of other unstable modes. Also, the modeling of Section 5 includes nonlinear coupling between multiple acoustic modes. Finally, as shown in Figure 7.1, a spectrum of pressure oscillations taken from the Caltech Jet Propulsion Center laboratory combustor reveals peaks associated with at least two acoustic modes of the system. The combustor has a rearward-facing step flameholder and burns a premixed methane/air flame with a power based on the higher heating value of about $45 \mathrm{~kW}$. Details of the laboratory apparatus were discussed in [33].

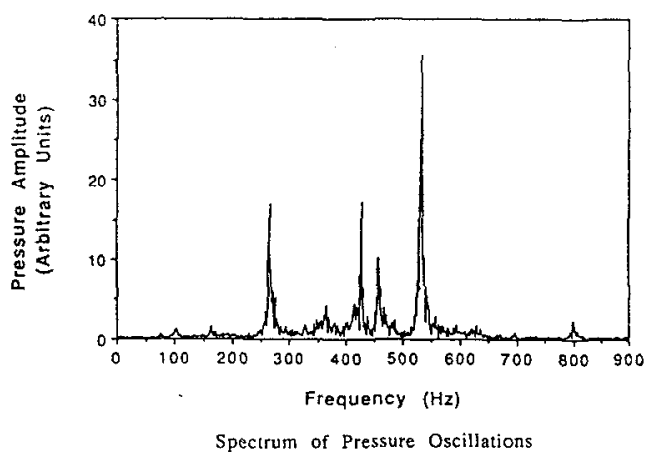

Figure 7.1

In addition to the fundamental frequency associated with each mode, system nonlinearities often result in spectra with both harmonic and subharmonic content. For some chamber geometries, the acoustic modes given by the equations $(2.13 a, b)$ will have wavenumbers that are not integer multiples of one another. In this case, frequencies associated with sums and differences of the modal frequencies may appear as well. Thus, active control schemes must address multiple modes that are known to display complex nonlinear behavior. How many modes must be considered?

A clue may be found by observing the spectra and looking for peaks at modal frequencies. However, the nonlinear dynamics of even a single oscillator (mode) can provide spectra with many peaks or spectra with broadband behavior characteristic of chaotic oscillations. A tool of modern dynamical systems theory may be used to characterize the minimum number of degrees-of-freedom required to describe an oscillating system. The "time-delay embedding method" allows a data signal to be placed or "embedded" as a phase-space trajectory. If start-up 
transients have ceased, the trajectory traces out a set of points in phase-space that comprise an "attractor". The fractal dimension of this attracting set of points provides a lower limit on the number of degrees-of-freedom of the system. Details on both the embedding method and the dimension-finding algorithms can be found in [34]-[36].

The dimension is presented as a function of the amplitude or scale of the oscillations. For very small scales, the experimental noise appears as an infinite dimensional dynamical system so that the dimension is equal to the embedding dimension. For scales larger than the largest amplitude of the oscillations, the attractor appears to be a point of dimension zero. Thus, the dimension must go from a high value to zero as the scale increases. If there exists a significant plateau, then the deterministic dynamics may be characterized by a single dimension. This plateau represents a system where the deterministic dynamics have significant "scale separation" from the noise.

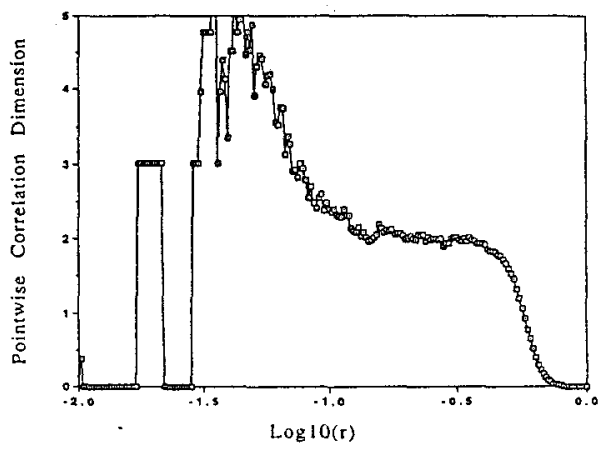

Pointwise Correlation Dimension

Figure 7.2

Figure 7.2 shows the dimension of the signal that was used to generate the spectrum of Figure 7.1. The number of points on the attractor was 8150 , which were taken at 80-microsecond intervals and embedded in a 9-D phase space using a 400-microsecond ( 5 points) time delay. This particular case reveals significant scale separation between noise and deterministic dynamics. A two-dimensional attractor is observed over approximately an order of magnitude of scales. The value of two is a result of the peaks in the spectrum at around $460 \mathrm{~Hz}$ and $530 \mathrm{~Hz}$. These frequencies were determined to correspond to linear acoustic modes of the system. Other peaks in the spectrum are subharmonics of these fundamental modes. Thus, two modes are observed to participate in quasiperiodic oscillations of a nonlinear nature. It should be noted that if multiple modes are "locked-in" to provide a periodic oscillation as in Section 5 , then the attractor dimension is unity. Thus, the dimension determination only provides a lower bound on the number of modes that participate in the oscillations.

The techniques presented above provide a methodology for determination of whether the oscillations are low-dimensional or whether too many degrees-of-freedom are present for deterministic models to be used. If there is not significant scale separation between the noise and the deterministic dynamics then the energy transfer from the combustion to the acoustics may be dependent upon turbulent fluctuations that are not tightly coupled to the acoustics. In this case, stochastic models may be required and active control measures may prove difficult to implement. On the other hand, even signals with complex, broad-band spectra may have enough scale separation that deterministic modelling and active control can be performed.

\section{REFERENCES}

(1) Doyle, J. D et al., article appears in these proceedings.

[2] Culick, F. E. C., "Combustion Instabilities in Liquid-Fueled Propulsion Systems," Paper No. 1, AGARD Conference Proceedings No. 450, October 1988.

[3] Culick, F. E. C., "Combustion Instabilities in Propulsion Systems," ASME Winter Meeting, December 1989.

[4] Culick, F. E. C. and Yang, V., "Prediction of the Unsteady Motions in Solid Propellant Rocket Motors," to appear in Nonsteady Burning and Combustion Stability of Solid Propellants, Progress in Astronautics and Aeronautics, (L. de Luca and M. Summerfield, Eds.).

[5] Culick, F. E. C., "Some Recent Results for Nonlinear Acoustics in Combustion Chambers," AIAA 13th Aeroacoustics Conference, Paper No. AIAA 90-3927, October 1990.

[6] Chu, B. T. and Kovasznay, L. C. G., "Non-linear Interactions in a Viscous Heat-Conducting Compressible Gas," $J$. Fluid Mech., Vol. 3, No. 5, pp. 494-512, 1957.

[7] Harrje, D. J. and Reardon, F. H. (Ed.) Liquid Propellant Rocket Instability, NASA SP-194, 1972.

[8] Culick, F. E. C., "Combustion Instability in Solid Rocket Motors, Volume II: A Guide for Motor Designers," C.P.I.A. Publication 290, 1981.

[9] Nickerson, G. R., Culick, F. E. C., and Dang, L. P., "Standard Stability Prediction Method for Solid Rocket Motors," Air Force Rocket Propulsion Laboratory Report AFRPLTR-83-0117, 1983.

[10] Paparizos, L. and Culick, F. E. C., "The Two-Mode Approximation to Nonlinear Acoustics in Combustion Chambers: I. Exact Solution for Second Order Acoustics," Combustion Science and Technology, Vol. 65, pp. 39-65, 1989.

[11] Tsien, H. S., "The Transfer Functions of Rocket Nozzles," J. American Rocket Society, Vol. 22, pp. 139-143, 1952.

[12] Schadow, K. C. et al., "Large-Scale Coherent Structures as Drivers of Combustion Instabilities," AIAA Paper No. 871326, July 1987.

[13] Sterling, J. D. and Zukoski, E. E., "Longitudinal Mode Combustion Instabilities in a Dump Combustor," AIAA Paper No. 87-0220, January 1987.

[14] Yu, K. et al., "Low Frequency Pressure Oscillations in a Model Ramjet Combustor-The Nature of Frequency Selection," AIAA Paper No. 89-0623, January 1987.

[15] Culick, F. E. C. and Schadow, K. C., "Pressure Oscillations in Ramjets," Proceedings of a JANNAF Combustion Subcommittee Workshop (C. Hudson, Ed.) C.P.I.A. Publication $535,1988$.

[16] Dines, P. J., Active Control of Flame Noise, PhD Thesis, Cambridge University, 1983.

[17] Heckle, M. A., Heat Sources in Acoustic Resonators, PhD Thesis, Cambridge University, 1985.

[18] Langhorne, P. J., "Reheat Buzz: An Acoustically Coupled Combustion Instability, Part I: Experiment"; "Part 2: Theory," (with G. J. Bloxsidge and A. P. Dowling), J. Fluid Mechanics, Vol. 193, pp. 417-473, 1988.

[19] Lang, W., Poinsot, T. and Candel, S. "Active Control of Combustion Instability," Combustion and Flame, Vol. 70, pp. 281-289, 1987.

[20] Poinsot, T., Bourienne, F., Candel, S. and Esposito, E. "Suppression of Combustion Instabilities by Active Control," J. Propulsion and Power, Vol. 5, No. 1, pp. 14-20, Jan.-Feb. 1989.

[21] Gulati, A: and Mani, R., "Active Control of Unsteady Combustion-Induced Oscillations," AIAA 28th Aerospace Sci- 
ences Meeting, Paper No. 90-0270, January 1990.

[22] Wilson, K. J., Gutmark, E., Schadow, K. C. and Smith, R. A., "Active Control of a Dump Combustor with Fuel Modulation," AIAA 29th Aerospace Sciences Meeting, Paper No. 91-0368, January 1991.

[23] Yang V., Sinha, A. and Fung, Y. T., "Linear Theory of Active Control of Pressure Oscillations in Combustion Chambers," to appear in J. Propulsion and Power, 1991.

[24] Franklin, G. F., Powell, J. D., and Emami-Naeini, A., Feedback Control of Dynamic Systems, Addison-Wesley Publishing Company, 1986.

[25] Yang, V., Kim, S., and Culick, R., "Triggering of Longitudinal Pressure Oscillations in Combustion Chambers I: Nonlinear Gasdynamics," Combustion Science and Technology, Vol. 72, pg. 183, 1990.

[26] Doedel, E. J. and Kernevez, J. P., "Software for Continuation Problems in Ordinary Differential Equations With Applications," preprint, California Institute of Technology, 1991.

[27] Lord Rayleigh, Theory of Sound, Vol. 2, Second Edition, p. 226, Dover, New York, 1945.

[28] Chu, B. T., "On Energy Transfer to Small Disturbances in a Viscous Compressible Heat-Conductive Medium," Johns Hopkins University, Air Force Office of Scientific Research, Contract AF 18(600)-1121, 1956.

[29] Culick, F. E. C., "A Note on Rayleigh's Criterion," Combustion Science and Technology, Vol. 56, pp. 159-166, 1987.

[30] Carrier, G. F., "The Mechanics of the Rijke Tube," Q. Applied Mathematics, Vol. 12, pp. 383-395, 1955.

[31] Merk, H. J., "Analysis of Heat-Driven Oscillations in Gas Flows I: General Considerations," Appl. Sci. Res., Vol. 6, Sec. A, pp. 317-335, 1957.

[32] Merk, H. J., "Analysis of Heat-Driven Oscillations in Gas Flows II: On the Mechanism of the Rijke-Tube Phenomenon," Appl. Sci. Res., Vol. 6, Sec. A, pp. 402-420, 1957.

[33] Sterling, J. D. and Zukoski, E. E., "Nonlinear Dynamics of Laboratory Combustor Pressure Oscillations," to appear in Combustion Science and Technology, 1991.

[34] Gershenfeld, N., "An Experimentalist's Introduction to the Observation of Dynamical Systems," chapter in Directions in Chaos, Vol. 2, Hao Bai-lin (Ed.), World Scientific Press, Singapore, 1988.

(35) Sterling, J. D., "Characterization of the Nonlinear Dynamics of Experimental Signals: Distinguishing Between Noise and Chaos," Caltech Series on Active Control of Combustion Instabilities, Document CI90-2, 1990.

[36] Sterling, J. D., "Dynamical Behavior of Combustion Instabilities," submitted for publication in Combustion Science and Technology, 1991.

\section{ACKNOWLEDGEMENTS}

This work was partially supported by the Office of Naval Research, Contract N00014-89-J-1753, the Air Force Office of Scientific Research, Grant No. 90-0188 and by Caltech funds. 